\title{
How Much do Needlestick Injuries Cost? A Systematic Review of the Economic Evaluations of Needlestick and Sharps Injuries Among Healthcare Personnel
}

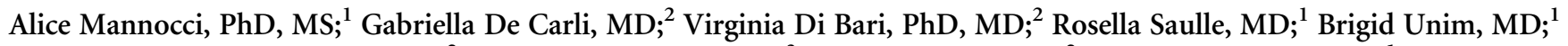 \\ Nicola Nicolotti, MD; ${ }^{2}$ Lorenzo Carbonari, PhD $;^{3}$ Vincenzo Puro, $\mathrm{MD} ;{ }^{2}$ Giuseppe La Torre, DSc ${ }^{1}$
}

ов JестіVE. To provide an overview of the economic aspects of needlestick and sharps injury (NSI) management among healthcare personnel (HCP) within a Health Technology Assessment project to evaluate the impact of safety-engineered devices on health care.

METHODs. A systematic review of economic analyses related to NSIs was performed in accordance with the PRISMA statement and by searching PubMed and Scopus databases (January 1997-February 2015). Mean costs were stratified by study approach (modeling or data driven) and type of cost (direct or indirect). Costs were evaluated using the CDC operative definition and converted to 2015 International US dollars (Int $\$$ ).

RESULTS. A total of 14 studies were retrieved: 8 data-driven studies and 6 modeling studies. Among them, 11 studies provided direct and indirect costs and 3 studies provided only direct costs. The median of the means for aggregate (direct + indirect) costs was Int $\$ 747$ (range, Int $\$ 199-$ Int $\$ 1,691$ ). The medians of the means for disaggregated costs were Int $\$ 425$ (range, Int $\$ 48-$ Int $\$ 1,516)$ for direct costs $(9$ studies) and Int $\$ 322$ (range, Int $\$ 152-$ Int $\$ 413$ ) for indirect costs (6 studies). When compared with data-driven studies, modeling studies had higher disaggregated and aggregated costs, but data-driven studies showed greater variability. Indirect costs were consistent between studies, mostly referring to lost productivity, while direct costs varied widely within and between studies according to source infectivity, HCP susceptibility, and post-exposure diagnostic and prophylactic protocols. Costs of treating infections were not included, and intangible costs could equal those associated with NSI medical evaluations.

CONCLUSIONS. NSIs generate significant direct, indirect, potential, and intangible costs, possibly increasing over time. Economic efforts directed at preventing occupational exposures and infections, including provision of safety-engineered devices, may be offset by the savings from a lower incidence of NSIs.

Infect Control Hosp Epidemiol 2016;37:635-646

Occupational exposures of healthcare personnel (HCP), especially nurses and surgeons, to bloodborne pathogens are frequent events in hospitals. ${ }^{1}$ In Italy every day, 300 HCP sustain an injury involving a contaminated needle or sharp medical device (needlestick and sharps injuries, NSIs), totaling $>100,000$ accidents per year, but only an estimated $45 \%$ are officially reported. ${ }^{2}$ Similar figures are available for the United States where, before legislation mandated widespread implementation of needlestickprevention devices incorporating a safety mechanism to cover the tip after use (safety-engineered devices, SEDs), 385,000 NSIs were estimated to occur annually, $60 \%$ of which were unreported. ${ }^{3}$ Available data from other European countries and all continents clearly demonstrate the high NSI burden worldwide. ${ }^{4-8}$

These NSIs have the potential to transmit virtually every pathogen present in human blood, either transiently or persistently. ${ }^{9,10}$ However, hepatitis B virus (HBV), hepatitis C virus (HCV), and human immunodeficiency virus (HIV), all bearing significant morbidity and mortality, together account for the vast majority of occupational infections reported in healthcare. In 2000, in the absence of interventions to prevent NSIs, these agents were estimated to have caused $>80,000$ new infections among HCP per year worldwide. ${ }^{11}$

Therefore, administrative, behavioral, and technical interventions aiming to reduce NSI frequency have been progressively introduced, including recommendations to prevent improper needle manipulations, ${ }^{12}$ education, ${ }^{13}$ provision of sharps containers for appropriate elimination of used devices, ${ }^{14}$ and SEDs. ${ }^{15,16}$ Each intervention has contributed to decreasing NSI frequency, but the best preventative strategy (ie, incorporating all these elements), requires significant

Affiliations: 1. Department of Public Health and Infectious Diseases, Sapienza University of Rome, Italy; 2. Department of Epidemiology, Pre-Clinical Research and Advanced Diagnostics, L. Spallanzani National Institute for Infectious Diseases, Rome, Italy; 3. Department of Economics and Finance \& CEIS, University of Rome "Tor Vergata" Italy.

Received November 10, 2015; accepted February 10, 2016; electronically published March 29, 2016

() 2016 by The Society for Healthcare Epidemiology of America. This is an Open Access article, distributed under the terms of the Creative Commons Attribution licence (http://creativecommons.org/licenses/by/4.0/), which permits unrestricted re-use, distribution, and reproduction in any medium, provided the original work is properly cited. All rights reserved. 0899-823X/2016/3706-0003. DOI: 10.1017/ice.2016.48 
investments of time, resources, and effort, which has limited their widespread implementation. ${ }^{17}$

Preventive interventions have therefore been included and have thus been reinforced in specific regulations regarding safety at work. In the United States, the Needlestick Safety and Prevention Act was passed in 2000, ${ }^{18}$ and the Occupational Safety and Health Administration endorsed the use of safe needles or needleless devices for the collection and/or withdrawal of body fluids and for the administration of fluids and medications. ${ }^{19,20}$ In Europe, the Council Directive 2010/ 32/EU, "Prevention from sharp injuries in the hospital and healthcare sector," fully in force since 2013, protects HCP from NSIs and their consequences, setting up integrated policies regarding risk assessment, risk prevention, training, education, and monitoring. Among the prevention measures, SEDs must be made available based on risk assessment, whereas $\mathrm{HBV}$ vaccination must be universally provided free of charge. Monitoring includes investigating the causes and circumstances of the accident and immediate care for the injured HCP that includes post-exposure prophylaxis (PEP), the necessary medical tests, health surveillance, and counseling where appropriate. Additionally, medical treatment is guaranteed. The economic impact of this directive is expected to be significant. ${ }^{21}$

Several studies have considered the economic burden of occupational NSIs, ${ }^{22-25}$ mostly providing direct costs (ie, postexposure management) and indirect costs (ie, counselling NSI victims, staff absence and compensation) from different perspectives and, more rarely, evaluating NSIs reduction after the introduction of preventive measures and the resulting economic impact. However, these studies vary widely in terms of setting, source of epidemiologic data, and considered costs, making it difficult to define a standardized cost for NSI management and prevention.

Given the increased costs of SEDs compared to conventional devices, in 2012, a Health Technology Assessment project was launched in Italy to evaluate this new technology and its overall impact on health care, including an economic analysis of costs deriving from NSIs and savings arising from their prevention. This systematic review represents the first part of this project.

\section{MATERIALS AND METHODS}

A systematic review of economic analyses of occupational NSIs among HCP was performed in accordance with the PRISMA statement. ${ }^{26}$ Independently, 2 authors consulted PubMed and Scopus databases and resolved eventual discrepancies by consulting a third author. The research algorithm used the following keywords: "cost, needlestick injuries" and "cost, occupational exposures, blood injuries." Inclusion criteria were language (English, Italian, French, or Spanish) and publication date (January 1997-February 2015).

The titles and abstracts of all articles were evaluated, eliminating duplicates. Articles were then assessed for eligibility through a full-text examination, and an article was included in the analysis if it provided an original estimate of either direct or indirect costs of managing a single NSI. The main stages of the systematic review are shown in Figure 1. Categorization and organization of the reference list were performed using JabRef 2.7.2 software.

Data extraction forms included author's name, publication year, country, source of the occurrence data, study period, population studied, NSI incidence, perspective, cost estimating approach (modeling or data-driven), items considered in cost, type of cost studied (direct and/or indirect), injury management cost (range), and currency equivalent.

To compare monetary values expressed in different currencies and for different years, the national inflation rates provided by the World Bank (http://data.worldbank.org/ indicator/fp.cpi.totl.zg) were used to express the injury management costs in national currencies 2015. These values were then converted to 2015 International US dollars (Int\$) using purchasing power parity (ppp) exchange rates (http://data. worldbank.org/indicator/pa.nus.ppp). To facilitate comparisons, all costs included in this review were converted to Int\$.

Data were analyzed by computing mean, median, and standard deviation (SD) as well as minimum and maximum

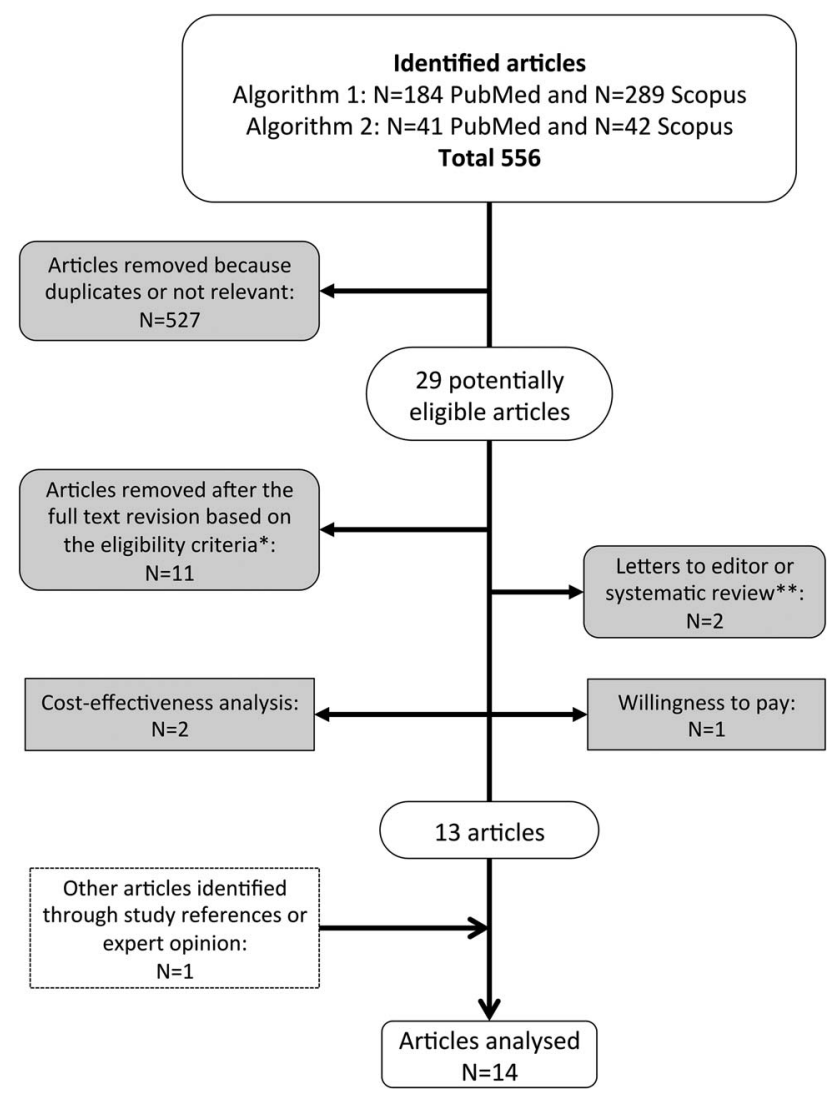

FIGURE 1. Flow-chart of the selection process. ${ }^{\star}$ Language other than English, Italian, French or Spanish, or published before January 1997. ${ }^{*}$ Study references were examined to identify other eligible studies. 
mean costs abstracted from each article. The weighted mean was used to account for the different proportion of resources used according to the type of event. The findings were stratified by study approach and type of cost (direct and/or indirect). All data used in this analysis were true cost data.

Direct and indirect costs of an NSI were evaluated using the CDC operative definitions:

1. Direct costs: Costs generally borne by a healthcare organization when an NSI occurs: baseline and follow-up laboratory testing, PEP, and other treatment eventually provided, including PEP side-effect management. According to which costs are borne by the organization, in certain circumstances, workers' compensation or the management of occupationally exposed HCP may be included with direct costs.

2. Indirect costs: These costs include time and wages diverted to receiving or providing exposure-related care: lost productivity associated with reporting and receiving initial and follow-up treatment for the exposure; healthcare provider time to evaluate and treat an employee; and healthcare provider time to evaluate and test the source, including obtaining informed consent for testing if applicable. For occupational health and infection control practitioners, these measures are part of their job responsibilities and therefore are not considered a diversion. Moreover, when staff absence and compensation are borne by a third-party payer, these may not be included with indirect costs. ${ }^{27}$

When analyzing in detail the items included within direct and indirect costs in each study, the authors originally assigned baseline and follow-up post-exposure visits differently according to the study context. However, when stratifying the study findings, the post-exposure visits, when disaggregated, were always considered as a direct cost and were assigned accordingly.

The quality of each study was evaluated using Drummond's checklist modified by La Torre et al. ${ }^{28}$

\section{RES U L T S}

In total, 14 studies from Europe, America, Asia, and Australia published from 1997 to 2013 met all the inclusion criteria. ${ }^{8,17,22,24,25,29-37}$ Their characteristics are reported in Tables 1 and 2.

\section{Cost Analysis}

Of the 14 studies estimating costs, 8 studies were data driven, while 6 studies used modeling (Table 3); 11 studies provided both direct and indirect costs (aggregated or disaggregated), while 3 studies only analyzed direct costs (Tables 2 and 3 ).

Costs were analyzed mainly from a hospital or university perspective in 7 studies; ${ }^{8,17,24,25,29,32,36} 3$ studies adopted the National Health System (NHS) perspective; ${ }^{22,31,33}$ and 1 study adopted both. ${ }^{30} \mathrm{~A}$ societal perspective was considered in 2 studies, ${ }^{34,35}$ and a national insurance perspective was considered in 1 study. ${ }^{37}$
Direct costs included testing the source and exposed HCP as well as post-exposure medical visits and treatment (ie, prophylaxis). Costs to treat an occupational infection were included in 2 studies only, which reported annual treatment $\operatorname{costs}^{32}$ and lifetime medical costs ${ }^{35}$ for HBV (Int $\$ 3,600$ and Int $\$ 31,306$, respectively), HCV (Int $\$ 24,424$ and Int $\$ 23,173$, respectively), and HIV infection (Int $\$ 35,745$ and Int $\$ 441,342$, respectively). These were excluded from the calculation of average NSI costs.

Indirect costs included lost productivity (time off work) associated with the time required for reporting and receiving initial and follow-up treatment as well as exposure consequences (eg, absence due to emotional distress and anxiety), overhead, compensation, and litigation.

Considering the 11 studies that provided aggregate direct + indirect costs, the overall median of the means of costs for managing an NSI was Int $\$ 747$ (mean of means, Int $\$ 861$; range of means, Int \$199- Int\$1691) (Tables 2 and 4).

The median of the means of the 9 studies that estimated disaggregated direct costs was Int $\$ 425$ (mean, Int $\$ 554$; range, Int $\$ 48-$ Int $\$ 1,516$ ), and the median of the means of the 6 studies that estimated disaggregated indirect costs was Int \$322 (mean, Int\$286; range, Int\$152-Int\$413).

Considering the type of approach, modeling studies had higher disaggregated and aggregated costs than data-driven studies: direct cost ratio, 3.4:1; indirect cost ratio, 1.84:1; combined direct + indirect cost ratio, 1.13:1. Costs from datadriven studies, however, showed greater variability, with standard deviations more than twice those of modeling studies (Table 4).

When estimating direct and indirect NSI management costs, the included cost items differed among studies (Table 5). Regarding direct costs, while laboratory tests represented the greater proportion in all studies, tests performed on the source and exposed HCP varied greatly. In total, 12 studies provided an itemized serological test menu. ${ }^{8,17,22,24,25,29-31,33,34,36,37}$ In all studies, investigators screened the source for antibodies against HCV (HCV-Ab) and HIV (HIV-Ab). HBV screening was performed mainly using surface antigen (HBsAg), ${ }^{8,24,25,29,30,31,34,36}$ adding surface antibody (HBsAb) ${ }^{22}$ or core antibody $(\mathrm{HBcAb})^{33,37}$ or through $\mathrm{HBcAb}$ alone. ${ }^{17}$

Regarding HCP, in 4 studies, ${ }^{17,29,30,33}$ HCV-Ab and HIV-Ab were always performed; in 6 studies, ${ }^{8,22,24,31,36,37}$ based on the source serostatus; and in 2 studies, ${ }^{25,34}$ the tests were based on HCP anxiety or other factor. HBV baseline screening varied; $\mathrm{HBsAb}$ was performed in vaccinated HCP regardless of the source, ${ }^{8}$ in case of a HBsAg-positive source, ${ }^{29}$ or following exposure to a positive or unknown source. ${ }^{22,24,30,37}$ In 1 study, unvaccinated $\mathrm{HCP}$, or vaccinated $\mathrm{HCP}$ with unknown or negative response, were tested for $\mathrm{HBs} \mathrm{Ag}, \mathrm{HBcAb}$, and $\mathrm{HBsAb}$ when exposed to an HBsAg-positive or unknown source. ${ }^{36}$ In another study, all HCP were tested only for $\mathrm{HBcAb} .{ }^{17}$ Finally, in 4 studies, HBV markers (usually $\mathrm{HBsAg}$ and $\mathrm{Ab}$ ) were performed regardless of the HCP vaccinal status or source serostatus. ${ }^{25,31,33,34}$ 
TAвLE 1. Characteristics of the Studies of Economic Analysis of Occupational Needlestick and Sharps Injuries (NSIs) Among Healthcare Personnel (HCP) Included in the Systematic Review, 1997-2013

\begin{tabular}{|c|c|c|c|c|c|c|}
\hline Country & Source of Occurrence Data & Study Period & Population & $\begin{array}{l}\text { Baseline NSI Incidence With } \\
\text { Conventional Devices }^{\mathrm{a}}\end{array}$ & $\begin{array}{l}\text { NSI Incidence After } \\
\text { Intervention }^{\mathrm{a}}\end{array}$ & $\begin{array}{c}\text { Study } \\
\text { (Reference) }\end{array}$ \\
\hline United States & Longitudinal surveillance & 2 y (June 1995-May 1997) & $\begin{array}{l}\text { HCP (hospital A: } \mathrm{CH} \\
\text { hospital B: } \mathrm{UH} \text { ) }\end{array}$ & $\begin{array}{l}\text { Hospital A: } 38.3 / 100 \text { beds per } \\
\text { year; hospital B: } 66 / 100 \text { beds } \\
\text { per year }\end{array}$ & Not applicable & 33 \\
\hline Spain & Longitudinal surveillance & 18 mo (January 1993-August 1995) & UH HCP & $8.2 / 100 \mathrm{HCP}$ per year & Not applicable & 37 \\
\hline France & Longitudinal surveillance & $\begin{array}{l}\text { Baseline: } 1 \text { y (1990); after: } 3 \text { y } \\
\quad(1995-1997)\end{array}$ & UH HCP & $12.7 / 100,000$ needles used & $\begin{array}{l}\text { 6.4/100,000 needles used } \\
\quad \text { (education } \\
\text { seminars + SEDs })\end{array}$ & 17 \\
\hline France & Longitudinal surveillance & 1 y $(2000)$ & UH HCP & 4.1/100 HCP per year & Not applicable & 36 \\
\hline United States & $\begin{array}{l}\text { Retrospective survey } \\
\quad \text { (recall) }\end{array}$ & 1 y (August 2003-August 2004) & $\mathrm{CH}$ nurses & $44.8 / 100$ nurses per year & Not applicable & 34 \\
\hline Spain & Longitudinal surveillance & 5 y (January 1998-December 2002) & UH HCP & 7.7/100 HCP per year & Not applicable & 22 \\
\hline Italy & $\begin{array}{l}\text { Longitudinal surveillance } \\
\text { and systematic review }\end{array}$ & $\begin{array}{l}75 \% \text { from national longitudinal } \\
\text { surveillance (2000-2003); } 25 \% \\
\text { from systematic review of literature } \\
(2002-2005)\end{array}$ & Hospital HCP & $\begin{array}{l}\text { Hospital A: 7.8/100,000 needles } \\
\text { used per year; hospital B: } \\
\text { 18.4/100,000 needles used } \\
\text { per year. }\end{array}$ & $\begin{array}{l}\text { Hospital A: } 0.9 / 100,000 \\
\text { needles used per year; } \\
\text { hospital B: } \\
\text { 2.2/100,000 needles used } \\
\text { per year (SEDs) }\end{array}$ & 30 \\
\hline Spain & Longitudinal surveillance & 1 y (March 2002-Februay 2003) & UH HCP & $19.4 / 100$ beds per year & $\begin{array}{l}\text { 7.2/100 beds per year (SEDs); } \\
\text { 3.2/100 beds per year } \\
\text { (SEDs + education and/or } \\
\text { change in procedure) }\end{array}$ & 29 \\
\hline United States & $\begin{array}{l}\text { Estimates from literature } \\
\text { data }\end{array}$ & 1 y $(1997-1998)$ & $\begin{array}{l}\text { Hospital and non- } \\
\text { hospital HCP }\end{array}$ & $\begin{array}{l}\text { 0.7/100 FTE in non-hospital } \\
\text { HCP; } 1.6 / 100 \text { FTE in hospital } \\
\text { HCP }\end{array}$ & Not applicable & 35 \\
\hline United States & $\begin{array}{l}\text { Opportunistic sample } \\
\text { from longitudinal } \\
\text { surveillance }\end{array}$ & 1 y $(2003)$ & HCP & Not applicable & Not applicable & 24 \\
\hline Sweden & Longitudinal surveillance & 1 y (2002) & HCP & $\begin{array}{l}3.14 / 100 \text { FTE per year }(1.89 \\
\text { with hollow-bore needles; } \\
60 \% \text { of the total })\end{array}$ & 1.1/100 FTE per year (SEDs) & 31 \\
\hline Chile & Longitudinal surveillance & 5 y $(2003-2007)$ & $\begin{array}{l}\text { University healthcare } \\
\text { students }\end{array}$ & $0.9 / 100$ students per year & Not applicable & 8 \\
\hline Belgium & $\begin{array}{l}\text { Estimates from literature } \\
\text { and market data }\end{array}$ & 1 y $(1999-2000)$ & $\mathrm{HCP}$ & $\begin{array}{l}\text { Injection needles used } \\
\quad 10.8 / 100,000 ; \\
\text { infusion therapy } 26.0 \text {; } \\
\text { insulin therapy } 23.5 \text {; } \\
\text { blood collection } 23.4\end{array}$ & $\begin{array}{l}\text { Injection needles used } 1.5 / \\
\quad 100,000 \text {; } \\
\text { infusion therapy } 8.08 \text {; } \\
\text { insulin therapy } 3.3 \text {; } \\
\text { blood collection } 7.0 \text { (SEDs) }\end{array}$ & 32 \\
\hline Korea & Longitudinal surveillance & 4 mo (October 2005-February 2006) & CH HCP & $\begin{array}{r}\text { 2.9/100 FTE per year or } \\
6.1 / 100 \text { beds per year }\end{array}$ & Not considered & 25 \\
\hline
\end{tabular}

NOTE. $\mathrm{CH}$, community hospital; FTE, full-time equivalent; HCP, healthcare personnel (doctors, nurses, technicians, students, etc.); NSIs, needlestick and sharps injuries; UH, university hospital; SEDs, safety-engineered devices.

${ }^{a}$ NSI incidence rates were standardized according to the available denominator (100 beds; 100 FTE or HCP; 100,000 used devices). 


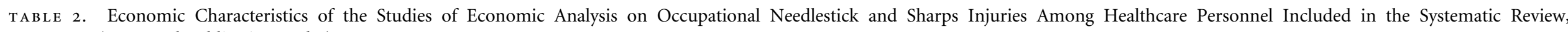
1997-2013 (Temporal Publication Order)

\begin{tabular}{|c|c|c|c|c|c|c|c|c|c|c|c|}
\hline Perspective & Approach & Determinants of Cost & Type of Cost ${ }^{\mathrm{a}}$ & $\begin{array}{l}\text { Costs } \\
\text { According } \\
\text { to the CDC } \\
\text { Definition }\end{array}$ & $\begin{array}{l}\text { Weighted Mean } \\
\text { Cost per Injury, } \\
\text { Int\$ (Range) }\end{array}$ & & Currency/Year & $\begin{array}{l}\text { Value per Injury, } \\
\text { Int } \$\left(\text { Range) }{ }^{c}\right.\end{array}$ & & $\begin{array}{l}\text { Comments on } \\
\text { Economic Results }\end{array}$ & $\begin{array}{l}\text { Study } \\
\text { (Reference) }\end{array}$ \\
\hline NHS & Data-driven & $\begin{array}{l}\text { Laboratory tests, } \\
\text { prophylaxis, lost } \\
\text { productivity (exposed } \\
\text { HCP and visiting staff) }\end{array}$ & Direct/indirect & Yes & $\begin{array}{l}\text { Direct }+ \text { indirect } \\
\text { Direct } \\
\text { Indirect }\end{array}$ & $\begin{array}{c}605(197-1,094) \\
473(\mathrm{NC}) \\
250(\mathrm{NC})\end{array}$ & US\$/1997 & $\begin{array}{l}\text { Direct + indirect } \\
\text { Direct } \\
\text { Indirect }\end{array}$ & $\begin{array}{c}893(290-1,614) \\
698 \\
369\end{array}$ & $\begin{array}{l}\text { Hospital A: } \$ 672 \\
\quad \text { (range: } \$ 340-1025) \text {; } \\
\text { Hospital B: } \$ 539 \\
\text { (range: } \$ 197-1,094)\end{array}$ & 33 \\
\hline $\begin{array}{l}\text { Third party } \\
\text { (Social } \\
\text { Security) }\end{array}$ & Modeling & $\begin{array}{l}\text { Laboratory tests, } \\
\text { prophylaxis, medical } \\
\text { visits, lost productivity } \\
\text { (exposed HCP, visiting } \\
\text { and administration } \\
\text { staff), overhead }\end{array}$ & Direct + indirect & $\cdots$ & Direct + indirect & $\begin{array}{c}39,564(23,074- \\
86,864)\end{array}$ & Pesetas/1994 & Direct + indirect & $648(378-1,423)$ & & 37 \\
\hline Hospital & Data driven & $\begin{array}{l}\text { Laboratory tests, } \\
\text { prophylaxis (including } 7 \\
\text { d off work), medical } \\
\text { visits, lost productivity } \\
\text { (exposed HCP and } \\
\text { visiting staff) }\end{array}$ & Direct + indirect & $\ldots$ & Direct + indirect & $325(\mathrm{NC})$ & US\$/1998 & Direct + indirect & 317 & $\begin{array}{l}\text { The study includes } \\
\text { (separately) the costs of } \\
\text { measures taken to reduce } \\
\text { injury rates. The cost- } \\
\text { effectiveness is } \$ 4,000 \text { per } \\
\text { injury prevented. }\end{array}$ & 17 \\
\hline Hospital & Data driven & $\begin{array}{l}\text { Laboratory tests, } \\
\text { prophylaxis, medical } \\
\text { visits, lost productivity } \\
\text { (exposed HCP) }\end{array}$ & $\begin{array}{l}\text { Direct/ } \\
\text { indirect }\end{array}$ & No & $\begin{array}{l}\text { Direct + indirect } \\
\text { Direct } \\
\text { Indirect }\end{array}$ & $\begin{array}{c}1,121 \\
1,005(\mathrm{NC}) \\
116(\mathrm{NC})\end{array}$ & Euro/2000 & $\begin{array}{l}\text { Direct + indirect } \\
\text { Direct } \\
\text { Indirect }\end{array}$ & $\begin{array}{c}1,691 \\
1,516 \\
175\end{array}$ & $\begin{array}{l}\text { Total occupational exposures } \\
\quad \text { cost in } 1 \text { year: } € 68,310 \text {; mean } \\
\quad=€ 281 ; \text { median }=€ 250\end{array}$ & 36 \\
\hline Societal & Data driven & $\begin{array}{l}\text { Laboratory tests, treatment, } \\
\text { medical visits, } \\
\text { medication, lost } \\
\text { productivity (exposed } \\
\text { HCP and visiting staff), } \\
\text { management of HIV PEP } \\
\text { side effects, emotional } \\
\text { distress and anxiety }\end{array}$ & Direct/indirect & No & $\begin{array}{l}\text { Direct + indirect } \\
\text { Direct } \\
\text { Indirect }\end{array}$ & $\begin{array}{c}159(145-201) \\
38(37-39) \\
121(107-163)\end{array}$ & US\$/2004 & $\begin{array}{l}\text { Direct + indirect } \\
\text { Direct } \\
\text { Indirect }\end{array}$ & $\begin{array}{c}199(182-252) \\
48(46-49) \\
152(134-204)\end{array}$ & $\begin{array}{l}\text { NSI management annual cost: } \\
\text { range: } \$ 25,896-\$ 36,066 . \\
\text { Direct + indirect costs per } \\
\text { injured nurse: mean }=\$ 259 ; \\
\text { (range, } \$ 235-\$ 328) ; \text { direct } \\
\text { costs per injured nurse: } \\
\text { mean }=\$ 113 \text { (range, } \$ 89.7- \\
\$ 182.2) \text {; indirect costs: mean } \\
=\$ 146 \text {. }\end{array}$ & 34 \\
\hline NHS & Modeling & $\begin{array}{l}\text { Laboratory tests, treatment, } \\
\text { medical visits, medical } \\
\text { instruments; lost } \\
\text { productivity (exposed } \\
\text { HCP and visiting staff), } \\
\text { overhead }\end{array}$ & Direct + indirect & NA & Direct + indirect & $388(172-1,502)$ & $\begin{array}{l}\text { Euro/ } \\
\quad 2002\end{array}$ & Direct + indirect & $714(317-2,765)$ & & 22 \\
\hline $\begin{array}{l}\text { Hospital } \\
\text { and } \\
\text { NHS }\end{array}$ & Modeling & $\begin{array}{l}\text { Laboratory tests, treatment, } \\
\text { medical visits, lost } \\
\text { productivity (exposed } \\
\text { HCP and visiting staff) }\end{array}$ & $\begin{array}{l}\text { Direct/ } \\
\text { indirect }\end{array}$ & Yes & $\begin{array}{l}\text { Direct }+ \text { indirect } \\
\text { Direct } \\
\text { Indirect }\end{array}$ & $\begin{array}{l}850(750-1,320) \\
586(516-910) \\
265(233-411)\end{array}$ & Euro/2005 & $\begin{array}{l}\text { Direct + indirect } \\
\text { Direct } \\
\text { Indirect }\end{array}$ & $\begin{array}{c}1,324(1,168-2,056) \\
913(804-1,417) \\
413(363-640)\end{array}$ & $\begin{array}{l}\text { NSI management annual } \\
\text { cost: } 36 \text { million euros }\end{array}$ & 30 \\
\hline Hospital & Data driven & $\begin{array}{l}\text { Laboratory tests, treatment, } \\
\text { nurse and medical visits }\end{array}$ & Direct + indirect & NA & Direct + indirect & 220 & Euro/2003 & Direct + indirect & 418 & $\begin{array}{l}\text { Cost-effectiveness for avoided } \\
\text { injury adopting SEDs: IV } \\
\text { catheters: } € 2.65 \text {; hypodermic } \\
\text { syringes: } € 869.79 \text {; butterfly } \\
\text { needles } € 1,195.99 \text {; needleless } \\
\text { administration sets } \\
€ 4,954.55 \text {; short IV catheters: } \\
€ 31,563.91 \text {. }\end{array}$ & 29 \\
\hline
\end{tabular}


Table 2. Continued

\begin{tabular}{|c|c|c|c|c|c|c|c|c|c|c|c|}
\hline Perspective & Approach & Determinants of Cost & Type of Cost ${ }^{\mathrm{a}}$ & $\begin{array}{l}\text { Costs } \\
\text { According } \\
\text { to the CDC } \\
\text { Definition }^{\text {b }}\end{array}$ & $\begin{array}{l}\text { Weighted Mean } \\
\text { Cost per Injury, } \\
\text { Int\$ (Range) }\end{array}$ & & Currency/Year & $\begin{array}{l}\text { Value per Injury, } \\
\text { Int\$ (Range) }\end{array}$ & & $\begin{array}{l}\text { Comments on } \\
\text { Economic Results }\end{array}$ & $\begin{array}{l}\text { Study } \\
\text { (Reference) }\end{array}$ \\
\hline Societal & Modeling & $\begin{array}{l}\text { Laboratory tests, treatment, } \\
\text { medical visits, lost } \\
\text { productivity (exposed } \\
\text { HCP) }\end{array}$ & Direct/indirect & Yes & $\begin{array}{l}\text { Direct }+ \text { indirect } \\
\text { Direct } \\
\text { Indirect }\end{array}$ & $\begin{array}{l}596 \\
339 \\
257\end{array}$ & US\$/2004 & $\begin{array}{l}\text { Direct + indirect } \\
\text { Direct } \\
\text { Indirect }\end{array}$ & $\begin{array}{l}747 \\
425 \\
322\end{array}$ & $\begin{array}{l}\text { National annual costs: tests: } \\
\$ 103,125,746 ; \text { lost } \\
\text { productivity: } \$ 81,187,457 \text {; } \\
\text { subsequent infections: } \\
\$ 4,186,548 \\
\text { Total cost for injuries: } \\
\$ 188,499,751\end{array}$ & 35 \\
\hline Hospital & Data driven & $\begin{array}{l}\text { Laboratory tests, treatment, } \\
\text { lost productivity } \\
\text { (exposed HCP and } \\
\text { visiting staff), additional } \\
\text { wages for management } \\
\text { of HIV PEP side effects }\end{array}$ & Direct + indirect & NA & Direct + indirect & $1,161(71-4,838)$ & US\$/2003 & Direct + indirect & $1,470(90-6,127)$ & $\begin{array}{l}\text { Mean cost related to the } \\
\text { infectious status of the } \\
\text { source:- } \$ 2,456 \text { if positive for } \\
\text { HIV (HBV, HCV included); } \\
\$ 650 \text { if positive for HCV; } \\
\$ 376 \text { for not infected/ } \\
\text { unknown source }\end{array}$ & 24 \\
\hline NHS & Modeling & $\begin{array}{l}\text { Laboratory tests, treatment, } \\
\text { medical visits }\end{array}$ & Direct & Yes & Direct & 2,513 & $\begin{array}{l}\text { Swedish kronor } \\
(\text { SEK)/2007 }\end{array}$ & Direct & 294 & $\begin{array}{l}\text { Adopting safety devices reduces } \\
\text { annual cost by } € 843,426 \text {. }\end{array}$ & 31 \\
\hline $\begin{array}{l}\text { University } \\
\quad \text { (training } \\
\text { school) }\end{array}$ & Data driven & $\begin{array}{l}\text { Laboratory tests; treatment; } \\
\text { medical visits }\end{array}$ & Direct & Yes & Direct & 149 & US\$/2007 & Direct & 170 & & 8 \\
\hline Hospital & Data driven & $\begin{array}{l}\text { Laboratory tests, treatment, } \\
\text { medical visits, surgical } \\
\text { treatment, lost } \\
\text { productivity (exposed } \\
\text { HCP) }\end{array}$ & Direct & Yes & Direct & $\begin{array}{l}\text { 119,673 Korean } \\
\text { won }(\$ 125)\end{array}$ & $\begin{array}{l}\text { Korean won } \\
\text { and US } \\
\text { dollar } \\
(955: 1) / 2006\end{array}$ & Direct & 173 & $\begin{array}{l}\text { Laboratory tests account for } \\
52.6 \% \text { of the cost }(45.9 \% \text { for } \\
\text { the HCP). Mean NSI } \\
\text { management cost is higher in } \\
\text { Seoul ( } \$ 139) \text { vs the suburbs } \\
(\$ 80) \text {. Regarding indirect } \\
\text { costs, there were no lost } \\
\text { working days among the } \\
\text { exposed HCP in the study. }\end{array}$ & 25 \\
\hline
\end{tabular}

NOTE. Int\$, 2015 International US dollars; HBV, hepatitis B virus; HCP, healthcare personnel; HCV, hepatitis C virus; HIV, human immunodeficiency virus; i.v., intravenous; NA, not applicable; NC, not computable; NHS, National Health System; NSI, needlestick and sharps injury; PEP: post-exposure prophylaxis; SEDs, safety-engineered devices.

${ }^{\mathrm{a} D i r e c t}+$ indirect: aggregated costs; direct/indirect: disaggregated costs.

${ }^{b}$ Data on categorization of the costs adapted according to Centers for Disease Control (CDC): direct costs include laboratory tests, treatment, medical visits; indirect costs include lost productivity, time-off productivity.

'Injury management costs were expressed in national currencies of 2015 using the national inflation rates provided by the World Bank (http://data.worldbank.org/indicator/fp.cpi.totl.zg), then these were converted to 2015 International US\$ (Int\$) using purchasing power parity (ppp) exchange rates (http://data.worldbank.org/indicator/pa.nus.ppp). 
Furthermore, in a single study, the source and HCP were also screened for syphilis, and in some cases, the source was screened for hepatitis A virus. These tests account respectively for $13.4 \%$ and $1.7 \%$ of the total tests performed in the study period. ${ }^{25}$

The antiretroviral HIV PEP regimen was specified in 8 of 13 studies. In 6 studies conducted between 1990 and 2003, a zidovudine-based regimen was adopted alone (in 1 study) ${ }^{34}$ or in combination with lamivudine, including a protease inhibitor according to risk (indinavir in 3 studies; ${ }^{17,22,33}$ nelfinavir in 2 studies $\left.^{29,36}\right)$. In 2 more recent modeling studies (2006 and 2013), a protease inhibitor-based regimen with lopinavir/ritonavir was considered, adding zidovudine/lamivudine $^{30}$ or tenofovir/emtricitabine ${ }^{32}$ (Table 5).

Finally, administration of hepatitis B immune globulin (HBIG) and HBV vaccination were included in the direct costs of an NSI in eight ${ }^{22,24,25,29,30,33,35,37}$ and seven ${ }^{24,25,30,33,35}$ studies, respectively (Table 5); in a single hospital study, HBV vaccination was administered but not included in the direct costs because the charges were borne by the NHS. ${ }^{29}$

Regarding indirect costs, 10 studies ${ }^{17,22,24,30,32-37}$ included lost productivity of an exposed HCP (eg, for initial reporting and treatment, follow-up appointments, and additional work

table 3. Distribution of Studies of Economic Analysis on Occupational Needlestick and Sharps Injuries Among Healthcare Personnel According to Type of Provided Costs and Study Approach

\begin{tabular}{llcc}
\hline & & \multicolumn{2}{c}{ Type of Approach } \\
\cline { 3 - 4 } $\begin{array}{l}\text { No. of } \\
\text { studies }\end{array}$ & Type of Cost & $\begin{array}{c}\text { Data } \\
\text { Driven }\end{array}$ & Modeling \\
\hline 3 & $\begin{array}{c}\text { Direct costs } \\
\text { Direct/indirect costs } \\
\text { (disaggregated) } \\
\text { Direct + indirect costs } \\
\quad \text { (aggregated) }\end{array}$ & 2 & 1 \\
5 & Total & 3 & 3 \\
14 & & 8 & 6 \\
\hline
\end{tabular}

time missed due to PEP side effects); 1 study $^{32}$ included also compensation and litigation costs (Table 5).

\section{Quality of the Studies}

The quality of the studies is presented in Table 6. For all investigations, the research question (item \#1), economic importance of the research question (\#2), viewpoints of the analysis (\#3), primary outcome measures for the economic evaluation (\#11), time horizon of costs and benefits (\#22), and conclusions from the data reported (\#34) were stated.

Three studies presented the incremental analysis (\#31), provided details of the design and results of effectiveness (\#9), and used a synthesis method or meta-analysis of estimates (\#10): the data-driven study by Armadans Gil, which had the highest quality score $(97 \%),{ }^{29}$ and 2 modeling studies, Leigh $(96 \%)^{35}$ and Hanmore $(94 \%)^{32}$ These 3 studies reported very different values of aggregate direct + indirect costs, with means of Int $\$ 418$, Int $\$ 747$, and Int $\$ 1,049$, respectively.

\section{I SCUSSION}

The overall aggregate direct + indirect costs for managing an NSI ranged from Int $\$ 650$ to Int $\$ 750$, considering the median and mean value of the mean costs borne by the hospital or NHS to manage exposures with different scenarios. These values were derived from both modeling and data-driven studies conducted across different countries and continents over a period of approximately 20 years.

Indirect costs are relatively consistent between studies; they mostly refer to lost productivity, which is usually calculated in minutes spent in baseline and follow-up visits by the exposed HCP and more rarely on days of staff absence; the median and mean values of the mean indirect costs ranged between Int $\$ 175$ and Int\$350.

Direct costs vary. The wide range of possible scenarios regarding the infectivity of the source, the susceptibility of the

тAвLE 4. Description of the Distribution of the Means of the Costs for Managing a Single Percutaneous Injury (2015 International US Dollars)

\begin{tabular}{|c|c|c|c|c|c|c|c|}
\hline Approach & Type of Cost & No. of Studies & \multicolumn{5}{|c|}{ Means of the Costs for Managing a Single NSI } \\
\hline \multirow{2}{*}{$\begin{array}{l}\text { Data driven } \\
(\mathrm{N}=8)\end{array}$} & Direct & 5 & 173 & 521 & 610 & 48 & 1,516 \\
\hline & Direct + indirect & 6 & 656 & 831 & 630 & 199 & 1,691 \\
\hline $\begin{array}{l}\text { Modeling } \\
(\mathrm{N}=6)\end{array}$ & Direct & 4 & 586 & 595 & 285 & 294 & 913 \\
\hline \multirow[t]{3}{*}{ All $(\mathrm{N}=14)$} & Direct & 9 & 425 & 554 & 467 & 48 & 1,516 \\
\hline & Indirect & 6 & 322 & 286 & 117 & 152 & 413 \\
\hline & Direct + indirect & 11 & 747 & 861 & 482 & 199 & 1,691 \\
\hline
\end{tabular}

NOTE. NSI, needlestick and sharps injury. 


\begin{tabular}{|c|c|c|c|c|c|c|c|c|}
\hline \multicolumn{6}{|c|}{ Direct costs } & \multicolumn{2}{|c|}{ Indirect costs } & \multirow[t]{2}{*}{$\begin{array}{l}\text { Study } \\
\text { (Reference) }\end{array}$} \\
\hline Laboratory Tests & $\begin{array}{l}\text { Drugs } \\
\text { HIV-PEP }\end{array}$ & HBV Vaccine & HBIG & $\begin{array}{l}\text { Subsequent } \\
\text { Occupational Infection }\end{array}$ & $\begin{array}{l}\text { Post-Exposure } \\
\text { Counseling/visit }\end{array}$ & Lost Productivity & Others & \\
\hline $\begin{array}{l}\text { Detailed costs; decision } \\
\text { algorithm not specified }\end{array}$ & $\begin{array}{l}\mathrm{AZT}+3 \mathrm{TC}+\mathrm{IDV}(\mathrm{cost} \\
\quad \text { for a } 4 \text {-week treatment })\end{array}$ & $\begin{array}{l}\text { Cost for } 3 \text { doses and } \\
\text { booster dose; cost in } \\
\text { hospital A includes } \\
\text { blood test }\end{array}$ & $\begin{array}{l}\text { Hospital A: cost per } \\
\text { dose; hospital B: } \\
\text { cost for } 5 \text { doses }\end{array}$ & Not included & $\begin{array}{l}\text { Cost depending on } \\
\text { simple, moderate, } \\
\text { and extensive risk }\end{array}$ & $\begin{array}{l}\text { Hospital A: average; } \\
\text { hospital B: not } \\
\text { included }\end{array}$ & Not included & 33 \\
\hline $\begin{array}{l}\text { Detailed costs; baseline } \\
\text { screening for HBV of the } \\
\text { source always performed, } \\
\text { regardless of HCP vaccinal } \\
\text { status; baseline HCP } \\
\text { screening for HCV and HIV } \\
\text { depending on the serostatus } \\
\text { of the source }\end{array}$ & $\begin{array}{l}\text { Not performed (no HIV } \\
\text { exposures) }\end{array}$ & $\begin{array}{l}\text { Not included (charge } \\
\text { borne by NHS) }\end{array}$ & $\begin{array}{l}\text { Cost/dose included in } \\
\text { the "post- } \\
\text { exposure } \\
\text { counselling/visit" } \\
\text { cost }\end{array}$ & Not included & $\begin{array}{l}\text { Cost/min for physician, } \\
\text { nurse and support } \\
\text { staff }\end{array}$ & Cost $/ \mathrm{min}$ & Overheads & 37 \\
\hline $\begin{array}{l}\text { Non-detailed costs; decision } \\
\text { algorithm not specified; } \\
\text { baseline screening of the } \\
\text { source and HCP for HBV, } \\
\text { HCV and HIV is always } \\
\text { performed. }\end{array}$ & $\begin{array}{l}\text { AZT or ddI }+ \text { PI; } \\
\text { costs include an average } \\
\text { of } 14 \text { days of PEP and } \\
\text { tests to detect drug } \\
\text { toxicity and a week off } \\
\text { work }\end{array}$ & Not included & Not performed & Not included & Not included & $\begin{array}{l}\text { Only for those who } \\
\text { took HIV-PEP, } \\
\text { evaluated using } \\
\text { the average gross } \\
\text { salary for nurses } \\
\text { and included in } \\
\text { PEP cost }\end{array}$ & Not included & 17 \\
\hline $\begin{array}{l}\text { Detailed costs; source screening } \\
\text { for HBV depending on the } \\
\text { HBV serostatus of the HCP; } \\
\text { baseline HCP screening for } \\
\text { HCV and HIV depending on } \\
\text { the serostatus of the source }\end{array}$ & $\begin{array}{l}\text { Average cost. } \\
\text { AZT/3TC + NFV } \\
(1 \text { case switched to } \\
\text { d4T + ddI + IDV })\end{array}$ & Not performed (no HBV & exposure) & $\begin{array}{l}\text { No subsequent } \\
\text { occupational } \\
\text { infection }\end{array}$ & $\begin{array}{l}\text { Cost/injury for } \\
\text { physician, nurse, } \\
\text { consultant, and } \\
\text { support staff }\end{array}$ & Cost/injury & Not included & 36 \\
\hline $\begin{array}{l}\text { Detailed costs; decision } \\
\text { algorithm not specified }\end{array}$ & $\begin{array}{l}\text { AZT alone regimen } \\
\quad+1 \text { multidrug regimen }\end{array}$ & Not included & Not included & Not included & $\begin{array}{l}\text { Cost/injury for } \\
\text { employee health } \\
\text { department, primary } \\
\text { care physician, and } \\
\text { consultant visit }\end{array}$ & Average & Not included & 34 \\
\hline $\begin{array}{l}\text { Detailed costs; baseline } \\
\text { screening for HBV of the } \\
\text { source is always performed, } \\
\text { regardless of HCP vaccinal } \\
\text { status. Baseline HCP } \\
\text { screening for HCV and HIV } \\
\text { depending on the serostatus } \\
\text { of the source }\end{array}$ & $\begin{array}{l}\text { AZT }+3 \mathrm{TC}+\mathrm{IDV} \text { (cost } \\
\text { includes tests to detect } \\
\text { drug toxicity })\end{array}$ & $\begin{array}{l}\text { Not included (charge } \\
\text { borne by NHS) }\end{array}$ & $\begin{array}{l}\text { Cost/dose included in } \\
\text { the "post- } \\
\text { exposure } \\
\text { counselling/visit" } \\
\text { cost }\end{array}$ & Not included & $\begin{array}{l}\text { Cost/min for physician, } \\
\text { nurse, and support } \\
\text { staff }\end{array}$ & $\begin{array}{l}\text { Cost } / \text { min for } \\
\text { physician, nurse } \\
\text { and support staff }\end{array}$ & Overheads & 22 \\
\hline $\begin{array}{l}\text { Detailed costs; baseline } \\
\text { screening of source and HCP } \\
\text { and FU not explicitly } \\
\text { reported, but deriving from } \\
\text { available national guidelines }\end{array}$ & $\begin{array}{l}\mathrm{AZT} / 3 \mathrm{TC}+\mathrm{LPV} / \mathrm{r} \text { (cost for } \\
\text { a 4-wk treatment) }\end{array}$ & Cost/dose & Cost $/ 2$ doses & $\begin{array}{l}\text { Only } 24 \text {-wk treatment } \\
\text { for HCV infection }\end{array}$ & $\begin{array}{l}\text { Cost/min for employee } \\
\text { health department } \\
\text { and physician visit }\end{array}$ & $\begin{array}{l}\text { Cost/visit for } \\
\text { exposed doctor } \\
\text { and nurse }\end{array}$ & Not included & 30 \\
\hline $\begin{array}{l}\text { Detailed costs; baseline } \\
\text { screening of the HCP for } \\
\text { HCV and HIV is always } \\
\text { performed regardless of the } \\
\text { serostatus of the source; } \\
\text { baseline screening for HBV, } \\
\text { HCV and HIV of the source } \\
\text { is performed if unknown }\end{array}$ & $\begin{array}{l}\mathrm{AZT}+3 \mathrm{TC}+\mathrm{NFV}(\operatorname{cost} \\
\text { includes tests to detect } \\
\text { drug toxicity) }\end{array}$ & $\begin{array}{l}\text { Not included (charge } \\
\text { borne by NHS) }\end{array}$ & Cost/dose & Not included & $\begin{array}{l}\text { Cost/injury for } \\
\text { physician and nurse }\end{array}$ & Not included & Not included & 29 \\
\hline
\end{tabular}


Average cost; decision algorithm Regimen not specified; not specified average cost

Cost included both HBV and HIV-PEP (no

source patients mono-infected with HBV)

Detailed costs; baseline $\mathrm{HCP}$ screening for HBV, HCV, and HIV, depending on serostatus of the source

Detailed costs; baseline HCP screening for HBV, HCV, and HIV, depending on the serostatus of the source

Non-detailed costs; decision algorithm not specified

Average costs; no decision algorithm
Regimen not explicitly reported, but deriving from available national

guidelines; total cost for

egimen not specified; cost Cost/dose for a 4-wk treatment

4 we + LPV/r for

4 weeks;
low- and

high-risk NSIs, including

both HBV- and

HIV-PEP

Regimen not specified; average cost/injury

4 doses for
Not performed (no HBV exposure in unvaccinated $\mathrm{HCP}$ )

unvaccinated and 1

booster dose for

vaccinated $\mathrm{HCP}$;

average cost for low-

high-risk NSIs, and HIV-PEP

Average cost/injury (cost included both HBV Not included vaccine and $\mathrm{HBIG}$ )
Not included
Not included
Not included

Not included

Cost depending on Not included intensity of

follow-up, and

subsequent

chronic HBV,

chronic or acute

$\mathrm{HCV}$, or HIV

infection

Mean cost and range for Mean cost and

initial evaluation, range for initia

Not included

evaluation

$\begin{array}{ll}\text { exposure } & \text { evaluation, } \\ \text { management, and } & \text { exposure }\end{array}$

follow-up visits management,

and follow-up

Not included

Cost/visit for nurse infectious diseas specialist, an psychiatric

Total cost for initial

evaluation, exposure

Not included Not included

Not included

management,

follow-up, and
emergency

department visits

$\mathrm{HBV} / \mathrm{HCV} / \mathrm{HIV}$ and erage cost for low- and Compensation and litigation

high-risk NSIs

$$
\begin{array}{ll}
\text { Average cost/injury for } & \begin{array}{c}
\text { No lost productivity Not included } \\
\text { medical }
\end{array} \\
\text { among the } \\
\text { consultations and }
\end{array} \quad \begin{aligned}
& \text { exposed HCP } \\
&
\end{aligned}
$$

NOTE. 3TC, lamivudine; AZT, zidovudine; d4T, stavudine; ddI, didanosine; FU, follow-up; HBIG, hepatitis B immune globulin; HBV, hepatitis B virus; HCV, hepatitis C virus; HCP, healthcare personnel; HIV, human immunodeficiency virus; IDV, indinavir; LPV/r, lopinavir/ritonavir; NFV, nelfinavir; NHS, National Health Service; NSI, needlestick and sharps injury; PEP, post-exposure prophylaxis; PI: protease inhibitor; TDF/FTC: tenofovir/emtricitabine. 
тавLE 6. Quality Results of the Selected Studies of Economic Analysis on Occupational Needlestick and Sharps Injuries Among Healthcare Personnel, 1997-2013

\begin{tabular}{|c|c|c|c|c|}
\hline Author & Year & Total Score & Maximum Score $^{\mathrm{a}}$ & Quality \% \\
\hline Jagger $\mathrm{J}^{33}$ & 1998 & 74 & 107 & 69 \\
\hline Solano Bernad VM ${ }^{37}$ & 1998 & 96 & 113 & 85 \\
\hline Roudot-Thoraval $\mathrm{F}^{17}$ & 1999 & 62 & 107 & 58 \\
\hline Nidegger $\mathrm{D}^{36}$ & 2003 & 63 & 79 & 80 \\
\hline Lee $\mathrm{WC}^{34}$ & 2005 & 72 & 85 & 85 \\
\hline Solano $\mathrm{VM}^{22}$ & 2005 & 72 & 89 & 81 \\
\hline Cazzaniga $S^{30}$ & 2006 & 93 & 116 & 80 \\
\hline Armadans Gil L $\mathrm{L}^{29}$ & 2006 & 107 & 110 & 97 \\
\hline Leigh $\mathrm{JP}^{35}$ & 2007 & 82 & 85 & 96 \\
\hline O’Malley EM EM $^{24}$ & 2007 & 80 & 107 & 75 \\
\hline Glenngård $\mathrm{AH}^{31}$ & 2009 & 66 & 107 & 62 \\
\hline Fica $\mathrm{CA}^{8}$ & 2010 & 71 & 91 & 78 \\
\hline Hanmore $\mathrm{E}^{32}$ & 2013 & 106 & 113 & 94 \\
\hline Oh HS ${ }^{25}$ & 2013 & 65 & 79 & 82 \\
\hline
\end{tabular}

${ }^{\mathrm{a}}$ Maximum score represents the expected score if the study was conducted with optimal practices. Different Maximum scores were shown because different study designs were reviewed according to Drummond's scale. ${ }^{28}$

exposed HCP, and the post-exposure diagnostic and prophylactic protocol account for the differences in the average direct costs within and between considered studies, particularly when analyzing studies using a different approach. In modeling studies, the source and exposed HCP are tested according to an optimized protocol. Prophylaxes are provided to all susceptible $\mathrm{HCP}$, and those protocols are always considered to have been completed. All exposed HCP attend follow-up visits and testing. In real life, HCP anxiety can influence the choice of baseline and follow-up testing, or the source situation may be more complicated (eg, multiple possible sources, need for supplemental, confirmatory testing, etc.). Acceptance, adherence, and completion rate of prophylactic treatments may be suboptimal; regimens may vary and cause adverse reactions requiring additional interventions; and compliance with follow-up protocols may differ.

Moreover, regardless of the approach, treatment costs vary between studies: the standard antiretroviral PEP regimen changed significantly over time, including different combinations and newer, more tolerable and expensive drugs in recent studies. Variations in HBIG dosage among protocols increased costs up to 4 -fold, and diagnostic tests evolved over time. Two recent modeling studies considering direct and indirect costs and using newer antiretrovirals had a median cost of Int $\$ 1,187$ (range, Int $\$ 1,049-$ Int $\$ 1,324) ;{ }^{30,32}$ however, too few recent studies are available to define a real and current overall cost.

Notwithstanding all the observed differences, comparisons between studies also identified significant similarities. Therefore, considering the wide range of possible situations, settings, and behaviors covered in selected studies, this overall amount (including direct and indirect costs) should represent a reliable estimate of the actual average cost for managing an NSI. However, the wide range of observed values should be taken into due account to avoid under- or overestimating the economic costs of managing these incidents or the potential savings resulting from their prevention.

A cautious interpretation of these findings is warranted. The literature search may be incomplete; moreover, different periods, countries, and study designs were compared. Part of the heterogeneity due to the approach and type of estimated costs was controlled by stratifying the studies. Furthermore, weighted means were calculated where disaggregated costs were available. However, this was not possible for all the studies considered. The effect of this heterogeneity is evident in the high variability (SD of the mean and width of the range) of the estimates. In addition, Drummond's checklist was designed to include all possible aspects concerning economic evaluations, but in this review, different study designs were compared, and it was not possible to apply all items to all articles. To eliminate disparities between the studies, a quality score was calculated based on the items considered appropriate for each study.

Despite these issues, this review confirms that the economic impact of managing NSIs is not negligible, and our analysis provides clues about where to reduce costs.

Protocols should be optimized based on the source; HCP vaccination should increase, and the response should be appropriately evaluated post-vaccination and readily available in case of injury. This recommendation is clearly demonstrated in the high-quality study by Armadans Gil et al, ${ }^{29}$ where most exposures involved vaccinated HCP exposed to known sources who tested negative for HCV and HIV; the mean NSI management cost (direct + indirect) was Int $\$ 418$.

Additional strategies to reduce costs could include the following: using rapid HIV tests to assess the source serostatus, thus avoiding unnecessary PEP, tests, and anxiety for the 
exposed HCP, ${ }^{38}$ and implementing tests for the early detection of bloodborne infections, which can shorten follow-up periods (eg, HIV-Ab/Ag test) and allow for early diagnosis at a lower cost (eg, $\mathrm{HCV}$ core-antigen testing replacing HCV-RNA).

Even if none of the retrieved studies included HCV-RNA testing in post-exposure protocols, it is recommended as alternative strategy in the United States and is strongly supported in a cost-effectiveness analysis. HCV-RNA testing at 1 month, at an average cost of Int $\$ 242$ per exposure, leads to earlier detection of HCV transmission and lowers the risk of progression to chronic hepatitis. ${ }^{39}$ Consequently, NSI management costs could also increase, and even more in the future, if direct-acting antivirals for hepatitis $\mathrm{C}$ are used in PEP, which would add a significant cost to treating these frequent events.

Are these costs truly representative of this issue? The costs of treating an occupational infection were not included in the calculation of average NSI costs, nor were those of litigation and compensation. Bloodborne infections other than HBV, $\mathrm{HCV}$, or HIV were not considered, though many different pathogens can be, and have been, occasionally transmitted through an NSI. Most importantly, intangible costs arising from an NSI were not evaluated. The single willingness-to-pay analysis identified in the literature reported that the high median amount HCP were willing to pay to avoid a sharpsrelated injury were Int $\$ 828$ in the base case and Int $\$ 1,237$ when exposed to HIV or HCV. This finding suggests that the costs of intangible aspects of HCP injuries, such as anxiety and distress, could equal costs associated with the medical evaluation of these injuries. ${ }^{40}$ However, in this study, the median time from injury to interview was 3 days. Thus, the interviewed subjects had not yet experienced the impact of being at risk of developing a bloodborne infection or the effects on personal and family life, sexual relationships, reproductive plans, breastfeeding, or professional expectations, which would increase the amounts reported above.

In conclusion, NSIs generate significant direct, indirect, potential, and intangible costs. While the costs for their prevention may seem high in the beginning, ultimately they prove to be the opposite. The economic expenditures directed toward enhancing interventions to prevent occupational exposures and infections, including provision of SEDs, will likely decrease over time, while enhancing HCP perception of their own value and affecting the quality of the care they provide. As highlighted in the preamble of the EU Directive, "Health and safety of workers is paramount and is closely linked to the health of patients."

\section{ACKNOWLEDGMENTS}

Financial support: This work was supported by the Italian Ministry of Health (grant no. RF-2009-1530527: "Health technology assessment of needlestickprevention devices to enhance safety of health care workers") and Ricerca Corrente IRCCS. We are deeply grateful to Andrea Baker for her thoughtful editing of the manuscript.
Potential conflicts of interest: G.D.C., V.P., and V.D.B. have developed educational material for Becton Dickinson. All other authors report no conflicts of interest related to this article.

Address correspondence to Gabriella De Carli, MD, Infezioni Emergenti e Riemergenti e Centro di Riferimento AIDS, Department of Epidemiology, PreClinical Research and Advanced Diagnostics, L. Spallanzani National Institute for Infectious Diseases - IRCCS, Via Portuense 292, 00149, Rome, Italy (gabriella.decarli@inmi.it).

\section{REFERENCES}

1. Puro V, De Carli G, Petrosillo N, Ippolito G, Studio Italiano Rischio Occupazionale da HIV Group. Risk of exposure to bloodborne infection for Italian healthcare workers, by job category and work area. Infect Control Hosp Epidemiol 2001;22:206-210.

2. PHASE Study Group. Prevention of occupational exposure to biohazard resulting from accidental percutaneous injury (puncture, wound, cut) in the hospital and healthcare sector. Technical overview and recommendations for the transposition and implementation of European Council Directive 2010/32/EU in Italy, 2012.

3. Panlilio AL, Orelien JG, Srivastava PU, et al. EPINet Data Sharing Network. Estimate of the annual number of percutaneous injuries among hospital-based healthcare workers in the United States, 1997-1998. Infect Control Hosp Epidemiol 2004;25:556-562.

4. Venier AG, Vincent A, L'Heriteau F, et al. Surveillance of occupational blood and body fluid exposures among French healthcare workers in 2004. Infect Control Hosp Epidemiol 2007;28:1196-1201.

5. Salzer HJ, Hoenigl M, Kessler HH, et al. Lack of risk-awareness and reporting behavior towards HIV infection through needlestick injury among European medical students. Int J Hyg Environ Health 2011;214:407-410.

6. Elder A, Paterson C. Sharps injuries in UK health care: a review of injury rates, viral transmission and potential efficacy of safety devices. Occup Med (Lond) 2006;56:566-574.

7. Zhang M, Wang H, Miao J, Du X, Li T, Wu Z. Occupational exposure to blood and body fluids among health care workers in a general hospital, China. Am J Ind Med 2009;52:89-98.

8. Fica CA, Jemenao P MI, Ruiz R G, et al. Biological risk accidents among undergraduate healthcare students: five years experience. Rev Chilena Infectol 2010;27:34-39.

9. De Carli G, Abiteboul D, Puro V. The importance of implementing safe sharps practices in the laboratory setting in Europe. Biochem Med (Zagreb) 2014;24:45-56.

10. Tarantola A, Abiteboul D, Rachline A. Infection risks following accidental exposure to blood or body fluids in health care workers: a review of pathogens transmitted in published cases. Am J Infect Control 2006;34:367-375.

11. Prüss-Ustün A, Rapiti E, Hutin Y. Estimation of the global burden of disease attributable to contaminated sharps injuries among health-care workers. Am J Ind Med 2005;48:482-490.

12. Centers for Disease Control and Prevention. Recommendations for prevention of HIV transmission in health-care settings. Morb Mortal Wkly Rep 1987;36(Suppl2):1S-18S.

13. National Institute for Occupational Safety and Health. A curriculum guide for public-safety and emergency-response workers: prevention of transmission of human immunodeficiency virus and hepatitis B virus. Cincinnati, OH: DHHS Publication No. (NIOSH), 1989; pp. 89-108. 
14. Linnemann CC Jr, Cannon C, DeRonde M, Lanphear B. Effect of educational programs, rigid sharps containers, and universal precautions on reported needlestick injuries in healthcare workers. Infect Control Hosp Epidemiol 1991;12:214-219.

15. Lamontagne F, Abiteboul D, Lolom I, et al. Role of safetyengineered devices in preventing needlestick injuries in 32 French hospitals. Infect Control Hosp Epidemiol 2007;28:18-23.

16. Tosini W, Ciotti C, Goyer F, et al. Needlestick injury rates according to different types of safety-engineered devices: results of a French multicenter study. Infect Control Hosp Epidemiol 2010;31:402-407.

17. Roudot-Thoraval F, Montagne O, Schaeffer A, Dubreuil-Lemaire ML, Hachard D, Durand-Zaleski I. Costs and benefits of measures to prevent needlestick injuries in a university hospital. Infect Control Hosp Epidemiol 1999;20:614-617.

18. Needlestick Safety and Prevention Act of 2000. Publication No. 106-430, 114 Stat. 1901; November 6, 2000.

19. Occupational Safety and Health Administration (OSHA). Occupational exposure to bloodborne pathogens; needlesticks and other sharps injuries; Final rule. 29 CFR part 1910. Washington (DC): US Department of Labor; 2001.

20. Occupational Safety and Health Administration (OSHA). OSHA instruction CPL 202.69: enforcement procedures for the occupational exposure to bloodborne pathogens. Washington (DC): OSHA Directorate of Compliance Programs, 2001.

21. Council of the European Union. Council Directive 2010/32/EU of 10 May 2010 implementing the Framework Agreement on prevention from sharp injuries in the hospital and healthcare sector concluded by HOSPEEM and EPSU. Official Journal of the European Union. 2010 (June 1):L 134(53):66-72. Eur-Lex website. http://eur-lex.europa.eu/LexUriServ/LexUriServ.do?uri=OJ: L:2010:134:0066:0072:en:pdf. Published 2010. Accessed January $13,2015$.

22. Solano VM, Hernández MJ, Montes FJ, Arribas JL. Update of the cost of needlestick injuries in hospital healthcare personnel. Gac Sanit 2005;19:29-35.

23. Whitby M, McLaws ML, Slater K. Needlestick injuries in a major teaching hospital: the worthwhile effect of hospital-wide replacement of conventional hollow-bore needles. Am J Infect Control 2008;36:180-186.

24. O'Malley EM, Scott RD, Gayle J, et al. Costs of management of occupational exposures to blood and body fluids. Infect Control Hosp Epidemiol 2007;28:774-782.

25. Oh HS, Yoon Chang SW, Choi JS, Park ES, Jin HY. Costs of postexposure management of occupational sharps injuries in health care workers in the Republic of Korea. Am J Infect Control 2013;41:61-65.

26. Liberati A, Altman DG, Tetzlaff J, et al. The PRISMA statement for reporting systematic reviews and meta-analyses of studies that evaluate health care interventions: explanation and elaboration. Ital J Public Health 2009;6:354-391.
27. Workbook for Designing, Implementing and Evaluating a Sharps Injury Prevention Program, 2008. Centers for Disease Control and Prevention website. http://www.cdc.gov/sharpssafety/pdf/ sharpsworkbook_2008.pdf; Published 2008. Accessed January 13, 2015.

28. La Torre G, Nicolotti N, de Waure C, Ricciardi W. Development of a weighted scale to assess the quality of cost-effectiveness studies and an application to the economic evaluations of tetravalent HPV vaccine. J Public Health 2001;19:103-111.

29. Armadans Gil L, Fernández Cano MI, Albero Andrés I, et al. Safety-engineered devices to prevent percutaneous injuries: cost-effectiveness analysis on prevention of high-risk exposure. Gac Sanit 2006;20:374-381.

30. Cazzaniga S, De Carli G, Sossai D, Mazzei L, Puro V. Il costo delle ferite accidentali e l'impatto dei dispositivi di sicurezza per la prevenzione del rischio di puntura accidentale. Mecosan 2006;58:99-116.

31. Glenngård AH, Persson U. Costs associated with sharps injuries in the Swedish health care setting and potential cost savings from needle-stick prevention devices with needle and syringe. Scand J Infect Dis 2009;41:296-302.

32. Hanmore E, Maclaine G, Garin F, Alonso A, Leroy N, Ruff L. Economic benefits of safety-engineered sharp devices in Belgiuma budget impact model. BMC Health Serv Res 2013;13:489.

33. Jagger J, Bentley M, Juillet E. Direct cost of follow-up for percutaneous and mucocutaneous exposures to at-risk body fluids: data from two hospitals. Adverse Exposure Prevention 1998;3:25-34.

34. Lee WC, Nicklasson L, Cobden D, Chen E, Conway D, Pashos CL. Short-term economic impact associated with occupational needlestick injuries among acute care nurses. Curr Med Res Opin 2005;21:1915-1922.

35. Leigh JP, Gillen M, Franks P, et al. Costs of needlestick injuries and subsequent hepatitis and HIV infection. Curr Med Res Opin 2007;23:2093-2105.

36. Nidegger D, Castel O, Peltier MP. Assessing the cost of occupational exposures to blood, in a French university hospital. Med Mal Infect 2004;34:28-36.

37. Solano Bernad VM, Rubio Cebrián S, Hernández Navarrete MJ, et al. Costs of accidental punctures in hospital health personnel. Gac Sanit 1998;12:29-38.

38. U.S. Public Health Service. Updated U.S. Public Health Service guidelines for the management of occupational exposures to $\mathrm{HBV}, \mathrm{HCV}$, and HIV and recommendations for postexposure prophylaxis. MMWR Recomm Rep 2001;50:1-52.

39. Deuffic-Burban S, Abiteboul D, Lot F, Branger M, Bouvet E, Yazdanpanah Y. Costs and cost-effectiveness of different followup schedules for detection of occupational hepatitis $\mathrm{C}$ virus infection. Gut 2009;58:105-110.

40. Fisman DN, Mittleman MA, Sorock GS, Harris AD. Willingness to pay to avoid sharps-related injuries: a study in injured health care workers. Am J Infect Control 2002;30:283-287. 